\title{
Determination of Phytocomponents of Twenty-one Varieties of Smokeless Tobacco using Gas Chromatography-Mass Spectroscopy (GC-MS)
}

\author{
Hassan A. Alhazmi ${ }^{\mathrm{a}, \mathrm{b}, *}$ (iD) ${ }^{\S}$, Asaad Khalid ${ }^{\mathrm{b}}$, Shahnaz Sultana ${ }^{\mathrm{b}, \mathrm{c}}$, Siddig I. Abdelwahab ${ }^{\mathrm{b}}$, \\ Waquar Ahsan ${ }^{\mathrm{a}, \mathrm{b}}$, Magbool E. Oraiby ${ }^{\mathrm{d}}$ and Mohammad AI Bratty ${ }^{\mathrm{a}, \mathrm{b}}$ \\ ${ }^{a}$ Department of Pharmaceutical Chemistry, College of Pharmacy, Jazan University, P.O. Box 114, Postal Code 45142, Jazan, Saudi Arabia. \\ ${ }^{b}$ Smokeless Tobacco Research Group, Substance Abuse Research Centre, Jazan University, P.O. Box 114, Postal Code 45142, Jazan, Saudi Arabia. \\ ${ }^{c}$ Department of Pharmacognosy, College of Pharmacy, Jazan University, P.O. Box 114, Postal Code 45142, Jazan, Saudi Arabia. \\ ${ }^{d}$ Poison Control and Medical Forensic Chemistry Center, General Directorate of Health Affairs, Jazan, Saudi Arabia.
}

Received 14 July 2018, revised 11 December 2018, accepted 11 December 2018.

\begin{abstract}
Smokeless tobacco (ST) leaves are being consumed by millions in Middle Eastern countries including Saudi Arabia, Sudan and Yemen, where the locally manufactured form is called shammah. We intended to explore and compare the phytocomponents of 21 different varieties of shammah that are commonly available in different cities of Jazan province of Saudi Arabia using gas chromatography-mass spectroscopy (GC-MS). A total of 61 different constituents were tentatively identified in these samples including both hazardous and non-hazardous compounds. Solvents of different polarity, such as petroleum ether, chloroform, ethanol and water were used to prepare the extracts. Interestingly, a number of new and unusual constituents were identified in some samples. $N$-methoxycarbonyl-amphetamine was detected as $6.76 \%, 4.88 \%$ and $2.58 \%$ of total compounds in Khadrah, Arishi and Sudani shammah samples, respectively. Similarly, the presence of caffeine (0.44-1.41 \%) in Adani cold from Jazan city and Abu Arish and Adani hot shammah from Abu Arish suggested the possibility of adulteration. Extremely hazardous arsenic and benzopyran derivatives were present in Sudani shammah from Sabya in small but significant amounts. Furthermore, carcinogenic components were also detected in significant amounts which indicate a relation between ST amounts used and different types of cancer, especially oral cancer. Chemometric hierarchical cluster analysis (HCA) revealed the existence of sufficient differences between samples from different extracts based on the polarity and dendrograms.
\end{abstract}

KEYWORDS

Smokeless tobacco, shammah, GC-MS, phytocomponents, hierarchical cluster analysis, toxic components, carcinogenic components.

\section{Introduction}

Smokeless tobacco (ST) has over 300 million users worldwide, and most of these users are from South Asian and Middle Eastern countries. ${ }^{1}$ The use of ST is more prevalent in the southern part of Saudi Arabia including Jazan province due to its proximity to Yemen, where it is legal to use and trade. ST, locally known as shammah, is a form of chewing tobacco consisting of powdered tobacco leaves mixed with variety of additives and flavouring agents such as carbonate of lime, ash, black pepper and volatile oils. ${ }^{2}$ Generally, ST comprises the leaves of N. tabacum, N. rustica, N. glauca, or N. nepalensis. ${ }^{3,4}$

Shammah is an addictive substance that is generally taken orally and ingesting the saliva produced thereafter. It is associated with a range of adverse health issues, including cancer and cardiovascular diseases. This could be attributed to a number of harmful substances including at least 28 chemicals which are identified as potential carcinogens, among which nitrosamines are reported as the most harmful. ${ }^{5}$ ST products cause weight loss, increased blood urea and creatinine which cause serious degenerative alterations and lead to periportal fibrosis in liver and edematous and calcified changes in renal glomerulus. ${ }^{6}$

* To whom correspondence should be addressed.

E-mail: haalhazmi@jazanu.edu.sa / hasalhazmi@gmail.com
Aqueous extracts of ST affect the growth of some oral bacteria, by affecting their healthy ecological balance and cause oxidative stress by minor degradation pathway of nicotine in the bacterium into hydroxynicotine and cotinine $\mathrm{N}$-oxide. ${ }^{78}$ Impairment of sperm head morphology and total sperm count along with various oxidative stress led genotoxicity and germ cell toxicity were reported in animals treated with tobacco. ${ }^{9}$ Nicotine itself is not carcinogenic, but its biologically activated forms such as nicotine-derived nitrosamine ketone (NNK) and $N$-nitrosonornicotine $(\mathrm{NNN})$ are responsible for its carcinogenic effect. ${ }^{10}$ Moderate and reversible toxicity along with organ weight loss was shown in oesophagus, stomach, liver, kidney and lung. ${ }^{11}$ Presence of ammonia, benzo[a]pyrene, cadmium, nickel, nicotine, nitrate and tobacco specific nitrosamines increase the risk of probabilistic cancer. ${ }^{12}$ Tobacco consumption has also been reported to cause systematic stress in humans with increase in inflammation, apoptosis and red blood cell membrane damage. ${ }^{13}$

Presence of different metals such as arsenic and nickel in ST synergize the risk factors associated with oral cancer by inducing nuclear damage. ${ }^{14,15}$ Nicotine is a pale yellow to dark brown liquid present up to $2-8 \%$ of the dry mass of the tobacco leaves. Products such as 3-(methylnitrosamino)-proprionitrile, nitrosamines, and nicotine initiate the production of reactive oxygen 
species (ROS), eventually leading to fibroblast, DNA and RNA damage and oral carcinogenicity in tobacco consumers. The metabolic activation of nitrosamine in tobacco by cytochrome P450 enzymes lead to the formation of NNN, a major carcinogen, and micronuclei, which are indicators of genotoxicity. These effects further enhance DNA damage, which eventually lead to oral cancer. ${ }^{16}$

In spite of a number of scientific findings showing ST products containing hazardous toxic/carcinogenic components, shammah of Saudi Arabia have not been thoroughly investigated. Therefore, the aim of this investigation was to analyze the chemical constituents of locally manufactured shammah samples available in Jazan region of Saudi Arabia using an improved GC-MS technique. The assessment of chemical composition would highlight the potential toxic and carcinogenic compounds present in ST products and would provide clinicians and researchers valuable information regarding the potential health effects that may result from the use of these ST products. It would also support the efforts of social and regulatory agencies to fight the usage of ST on a scientific basis.

\section{Experimental}

\subsection{Shammah Material}

The powdered form of 21 samples of local shammah products were purchased in various areas of the Jazan region of Saudi Arabia. The purchased ST products represent the most widely used varieties covering all areas of Jazan province. These ST products were named by their local names followed by the site (city) of collection. Shammah samples called Khususi (Special) were collected from different manufacturers in Ahad al Masarihah (S1), Sabya (S2), Samtah (S3) and Abu Arish areas (S4). Khadrah (Green) samples were purchased in Ahad al Masarihah (S5), Sabya (S6), Jazan city (S7) and Abu Arish (S8). Arishi shammah samples were collected in Ahad al Masarihah (S9), Sabya (S10), Jazan city (S11), Samtah (S12) and Abu Arish (S13). Samples of Adani baarid (cold) shammah were collected in Ahad al Masarihah (S14), Sabya (S15), Jazan city (S16) and Abu Arish (S17), whereas, Adani haar (hot) samples were collected in Ahad al Masarihah (S18) and Abu Arish (S19). Sudani shammah (S20) and Suhail shammah (S21) were purchased from Sabya.

\subsection{Preparation of Different Extracts}

$0.2 \mathrm{~g}$ of each shammah sample was taken and homogenized at $5000 \mathrm{rpm}$ with $100 \mathrm{~mL}$ each of four different polar (water and ethanol) and non-polar (chloroform and petroleum ether) solvents thrice. After $24 \mathrm{~h}$, the combined extracts of each solvent were filtered using Whattman filter paper $(0.45 \mu)$ and concentrated by distillation and dried under reduced pressure to obtain dark coloured mass. The residue was stored at $4^{\circ} \mathrm{C}$ in the dark for GC-MS analysis.

\subsection{GC-MS Analysis of the Extract}

GC-MS analysis were carried out on a Shimadzu Gas Chromatograph (GC-2010, Germany) instrument fitted with a capillary column TR-5MS (Thermo Scientific TRACE 260F130P, Texas, U.S.A.) $(30 \mathrm{~m} \times 0.25 \mathrm{~mm})$ and film thickness $0.25 \mu \mathrm{m}$. The carrier gas helium (He) was used at a flow rate of $1.2 \mathrm{~mL} \mathrm{~min}^{-1}$. The initial temperature kept was $70{ }^{\circ} \mathrm{C}$ followed by heating at a rate of $15^{\circ} \mathrm{C}$ per minute to $290^{\circ} \mathrm{C}$, which was held for $30 \mathrm{~min}$. The chromatograph was coupled to a Shimadzu QP2010 Ultra MS detector with electron-ionization system and ionization energy of $70 \mathrm{eV}$. Ion source temperature was maintained at $230^{\circ} \mathrm{C}$.

\subsection{Identification of Constituents}

Most of the constituents were identified by GC-MS by comparing their retention indices with either the authentic standards (Sigma Aldrich, Darmstadt, Germany) available in the laboratory or with the retention indices which were in close agreement with reference. Further identification was achieved by GC-MS where the fragmentation patterns of mass spectra were compared with those stored in the spectrometer database using the NIST08 and Wiley 9 built-in libraries. The area\% was used to quantify the components present.

\subsection{Statistical Cluster Analysis}

Due to significant variations between the contents of various shammah samples, most abundant components from all samples were subjected to multivariate chemometric analysis. Hierarchical Cluster analysis (hca) was performed and dendrograms were developed for test samples using SPPS software version 22.0.

\section{Results}

Using GC-MS analysis, we were able to separate and identify various constituents of shammah samples efficiently. Fig. 1 shows representative chromatograms of three shammah samples. All components identified in shammah samples along with their retention times are summarized in Table S1 (supplementary material), whereas, toxic/carcinogenic components present in shammah samples, along with their maximum area\% detected in any extract, are listed in Table 1. Structures of some important constituents detected in various shammah samples are shown in Fig. 2. These represent the major toxic constituents that were present in more than one sample.

The GC-MS analysis showed that the petroleum ether, chloroform, ethanol and aqueous extracts of Khususi (Special) shammah samples S1, S2, S3 and S4 possessed various types of chemical constituents including alkanes, siloxanes, fatty acid ester and pyridine derivatives (Table S1). Nicotine or, 3-(1methyl-2-pyrrolidinyl)pyridine was present in highest area\% with $2.1-88.81 \%$ in various samples depending upon the extract with petroleum ether being the highest. Among siloxane derivatives, dodecamethyl cyclohexasiloxane was present as $0.04-4.12 \%$ in all the samples. 3-Isopropoxy-1,1,1,7,7,7-hexamethyl-3,5,5-tris(trimethylsiloxy)tetrasiloxane was present only in S1, S2 and S3 samples with highest area\% of $2.02 \%$ in ethanolic extract of S1. Other siloxanes, tetracosamethyl cyclododecasiloxane and hexadecamethyl heptasiloxane were present only in the aqueous extract of S1, which contained another siloxane, octadecamethyl cyclononasiloxane in significantly higher area\% (40.89\%). Several fatty acids and their esters were also found to be present in all Khususi (Special) samples in relatively low to higher area\% with methyl linoleate (5.64\%) and methyl oleate $(35.26 \%)$ present in all samples with highest area\% in aqueous extracts of S4 and S2, respectively. S1 showed presence of other fatty acid esters such as neopentyl hydroxyacetate and butyl 2-amino-5-methylbenzoate which were not present in other Khususi samples. On the other hand, methyl stearate was only present in S3 and S4 in traces but not present in $\mathrm{S} 1$ and S2. Alkanes such as undecane (S1-S4), tridecane (S1-S4), dodecane (S2), 2,6,10,14-tetramethyl heptadecane (S2), pentadecane (S1, S2) and tricosane (S2, S4), tetratriacontane (S3, S4) were present in low amounts in different extracts. 9-Octadecenamide and erucylamide were the only amides that were present merely in S4.

Khadrah (green) shammah S5, S6, S7 and S8 also possessed various types of chemical constituents including alkanes, silox- 

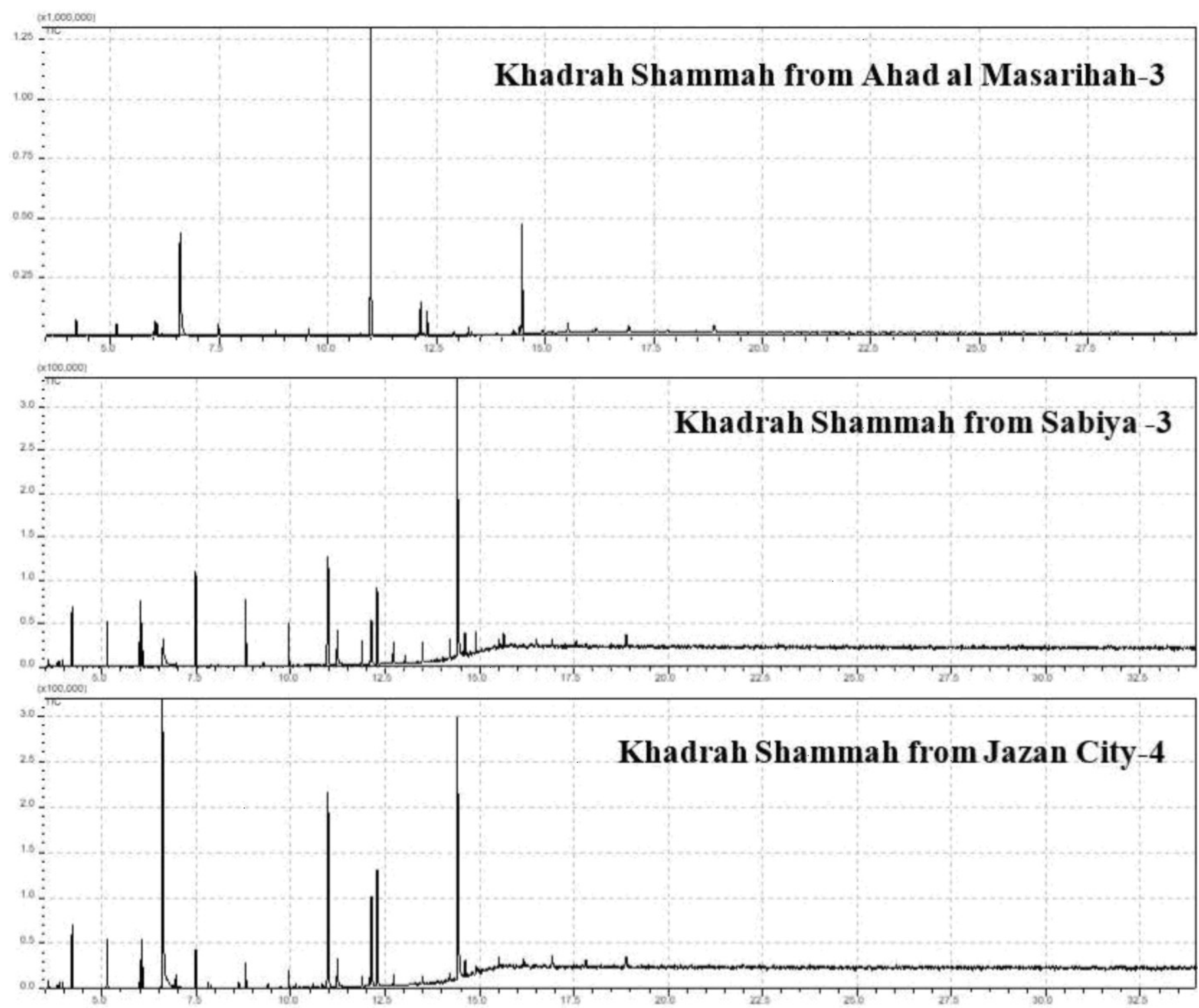

Figure 1 Representative of typical GC-MS total ion current (TIC) chromatograms of three shammah samples.

anes, pyridine derivatives and fatty acid esters. Nicotine was present in all the samples ranging from $1.75-89.37 \%$ of the total compounds, with maximum area $\%$ again observed in petroleum ether extract. The other pyridine derivative, $(1 \mathrm{~s}, 2 \mathrm{~s})$-nicotine- $\mathrm{N}$-oxide, was present in S5 and S7 only in the non-polar extracts. Among siloxanes, dodecamethyl cyclohexasiloxane (S5-S7), 3-isopropoxy-1,1,1,7,7,7-hexamethyl-3,5,5-tris(trimethylsiloxy)tetrasiloxane (S5, S6), 1,1,1,3,3,5,5-heptamethyltrisiloxane (S7) and hexamethyl cyclotrisiloxane (S6-S8) were the most important ones in all the extracts present in minor quantities. Several fatty acids and their esters were also present including, methyl hexadecanoate (S5-S8; 1.42-39.34 \%), methyl oleate (S5-S8; 1.43-36.92\%), methyl linoleate (S5-S7), methyl stearate (S5, S7) and methyl 9, 10-dichloro-octadecenoate (S5) in different samples majorly in non-polar solvents. Alkanes on the other hand were also present in low to good quantities among which undecane (S5-S7), tridecane (S6), pentadecane (S5) and tricosane (S7) were the major ones. 88 possessed a nitrile derivative, oleanitrile in aqueous extract $(13.73 \%)$ that was not present in other Khadrah samples. Several amines and amides were also found to be present including 13-docosenamide (S7, S8) and $\mathrm{N}$-methoxycarbonyl amphetamine (S8; $6.76 \%$ ).

The third variety of shammah tested was Arishi shammah (S9,
S10, S11, S12 and S13) which revealed the presence of nicotine, which was extracted mainly in the non-polar solvents petroleum ether and chloroform and the area $\%$ in different samples were found to be in the range of 4.52-89.45\%. Another important constituent, siloxane, included dodecamethyl cyclohexasiloxane (S9-S11; 0.86-3.52\%), 3-Isopropoxy-1,1,1,7,7,7-hexamethyl3,5,5-tris(trimethylsiloxy)tetrasiloxane (S9, S10, S13) and hexamethyl cyclotrisiloxane (S10, S11) which were detected in different sample extracts. Among alkanes, undecane (S9, S11-S13), tridecane $(\mathrm{S} 9, \mathrm{~S} 10)$, tricosane $(\mathrm{S} 9, \mathrm{~S} 11, \mathrm{~S} 12)$ were the important ones detected mainly in the non-polar solvents. The quantities of fatty acid and esters present were significantly high in almost all the Arishi samples. The major fatty acid esters included methyl hexadecanoate (S9-S13; 1.7-34.1 \%), methyl linoleate (S9-S13; 0.65-5.16\%), methyl 7-octadecenoate (S9) and methyl oleate (S9-S13; 0.41-25.52\%). Oleic acid, a fatty acid, was detected in S9 (1.41-3.07\%) only. S10 showed presence of the only aromatic ester, dimethyl 2-hydroxy-4-[(tetrahydropyran2-yl)oxy)methyl]bezene-1,3-dicarboxylate, nitrogenous heterocyclic compound, 1-tert-butyl-3,3,4-trimethyl-3,4-dihydro$1 H$-benzopyran-1-ol and the other aromatic compound 1-phenyl-5,5-dimethyl-4,6-dioxa-5-sila-8-nitrooct-1-ene in considerable amounts. S11 also showed the presence of aromatic 


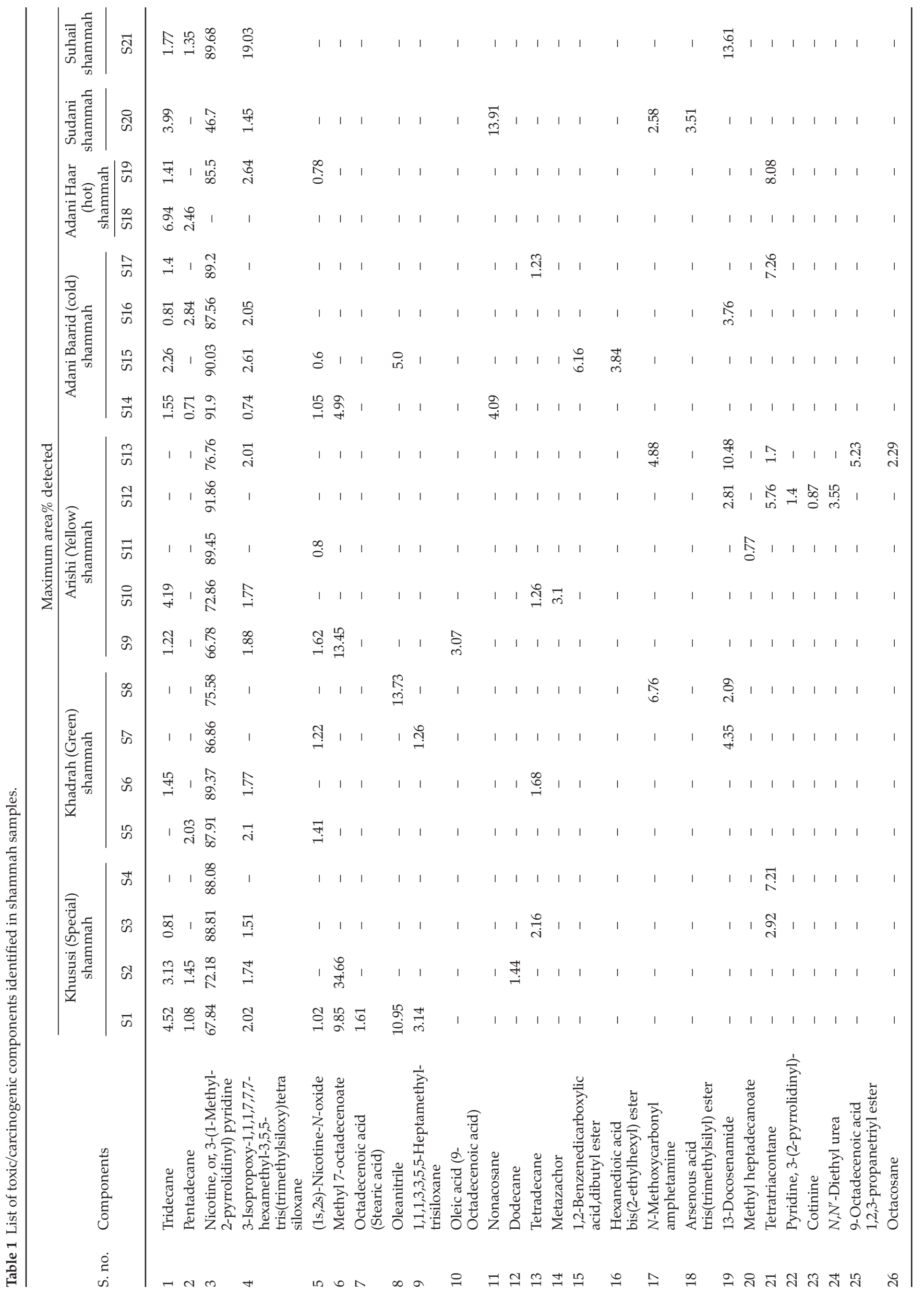




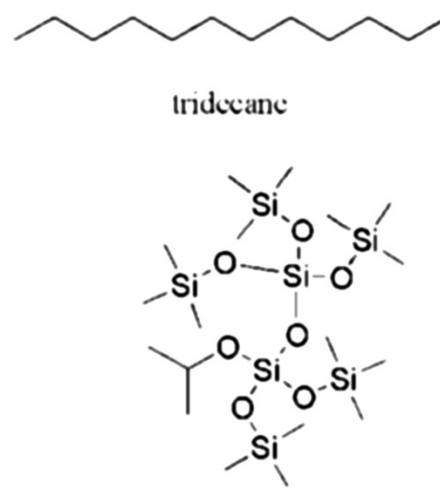

3-Isopropoxy-1,1,1,7,7,7-hexamethy3,5,5-tris(trimethylsiloxy)tetrasiloxane

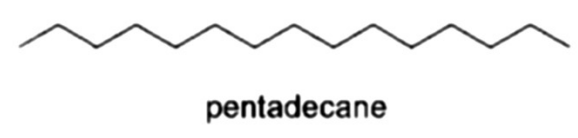

pentadecane

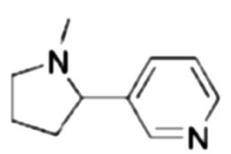

nicotine

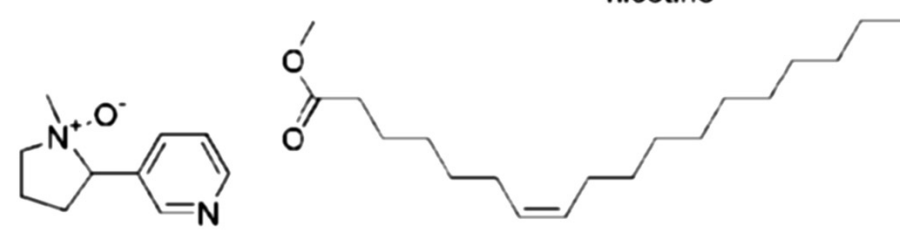

(1s,2s)-Nicotine-N-oxide

Methyl 7-octadecenoate<smiles>COC(=O)NC(C)Cc1ccccc1</smiles>

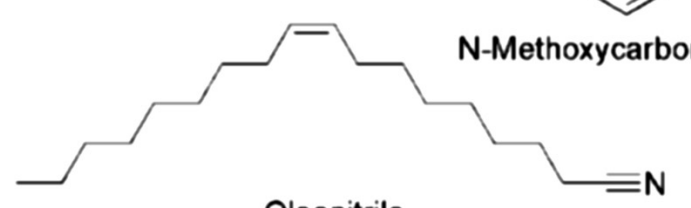

Oleonitrile

13-Docosenamide

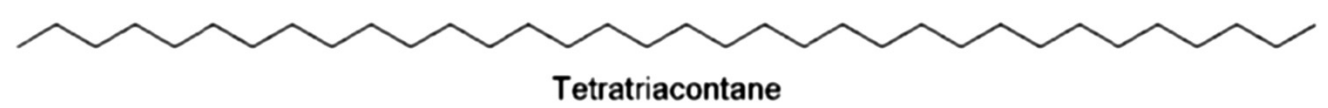

Figure 2 Structures of some important phytocomponents detected in various shammah samples.

ester, butyl 2-amino-5-methylbenzoate. 13-Docosenamide, an amide was detected in S12 (1.53-2.81 \%) and S13 (0.13-10.48 \%) only and not in other Arishi samples. $N, N^{\prime}$-diethyl urea was found to be present in S12 (3.55\%) only, whereas, S13 showed the presence of $\mathrm{N}$-methoxycarbonyl amphetamine $(4.88 \%)$ that was not observed in other Arishi samples.

Another variety called Adani baarid (cold) shammah (S14, S15, S16 and S17) showed the presence of two pyridine derivatives, nicotine and nicotine- $N$-oxide. Nicotine- $N$-oxide was not detected in S16 and S17 but was present in minor quantities $(0.29-1.05 \%)$ in S14 and S15. Nicotine was the major constituent having maximum percentage in all the samples in a range of $0.03-91.9 \%$. S14 revealed presence of several alkanes including undecane, tridecane, pentadecane, tricosane, pentatriacontane and nonacosane in minor quantities ranging from $0.23-4.09 \%$ in different extracts, whereas, S15 had only two alkanes tridecane and tricosane in trace quantities (1.5-2.26 \%). S16 and S17 also possessed all the alknaes that were present in S14 with additional tetradecane and tetracontane in minor quantities. Among siloxanes, dodecamethyl cyclohexasiloxane and 3-isopropoxy1,1,1,7,7,7-hexamethyl-3,5,5-tris(trimethylsiloxy)tetrasiloxane were the major contributors in all the samples. Several fatty acids and their esters were also observed in these samples including methyl palmitate (S14-S17), methyl 7-octadecenoate (S14) and methyl linoleate (S16) in minor to good quantities. The Adani Baarid shammah from Sabya (S15) showed the presence of aliphatic ester, hexanedioic acid bis(2-ethylhexyl) ester (3.84 \%) and oleanitrile (5\%), which were not present in other samples. 1,2-Benzenedicarboxylic acid dibutyl ester, 1,2-benzenedicarboxylic acid,bis(2-methylpropyl) ester, 1,2-benzenedicarboxylic acid bis(2-methoxyethyl) ester and 1,2-benzenedicarboxylic acid bis(2-ethylhexyl) ester were other aromatic ester derivatives present in minor to major quantities (1.85-45.29 \%).
S16 also showed the presence of an amide, 13-docosenamide $(0.15-3.76 \%)$, whereas, interestingly, caffeine was also identified in S16 (0.09-0.5\%) and S17 (0.68-1.41\%) samples suggesting the presence of additives such as tea leaves or coffee to enhance the organoleptic properties.

Adani Haar (hot) shammah (with cardamom) were also procured from two cities Ahad al Masarihah (S18) and Abu Arish (S19). Interestingly, S18 was devoid of nicotine and no peak corresponding to nicotine was detected. On the other hand, it was present as 65.55-85.5\% in S19 with additional nicotine- $N$-oxide present in minor quantity. Alkanes present in S18 were undecane, pentadecane, tridecane, octadecane and tricosane in minor quantities from $1.31-12.17 \%$, whereas, the major alkanes present in S19 were tridecane and octadecane (0.35-1.41 \%). Among siloxanes, dodecamethyl cyclohexasiloxane and 3-isopropoxy-1,1,1,7,7,7-hexamethyl-3,5,5tris(trimethylsiloxy)tetrasiloxane were the two major derivatives present in these samples. Methyl palmitate was the only major fatty acid ester present in S19. Interestingly, S19 also showed the presence of caffeine as an additional alkaloid present in different extracts in different quantities ranging from $1.31-2 \%$.

Sudani Shammah from Sabya (S20) showed nicotine in comparatively lesser quantity (21.83-46.7 \%). Among alkanes tridecane, urdecane and nonacosane were present in minor area\% from 1.19-13.91\% in different extracts. Butyl 2-amino-5methylbenzoate was the only aromatic ester (2.2-53\%), 1-phenyl-5,5-dimethyl-4,6-dioxa-5-sila-8-nitrooct-1-ene was the only phenyl derivative and 4-formyl (benzoyl)-isopropylamide was the only amide derivative present. Interestingly, S20 also showed the presence of $N$-methoxycarbonyl amphetamine in ethanolic extract $(2.58 \%)$ that signifies the presence of Khat leaves mixed with shammah leaves. Another important finding 

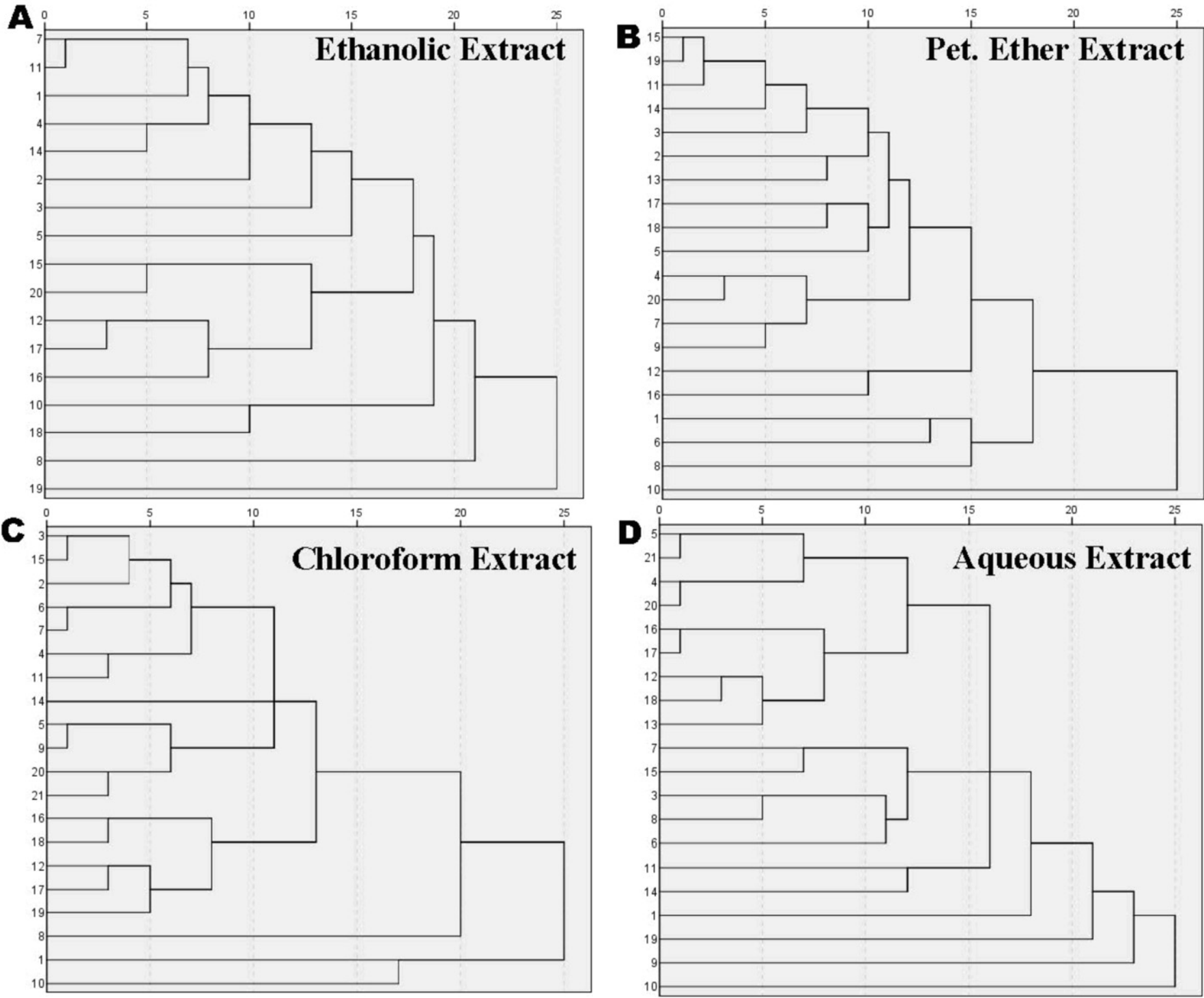

D

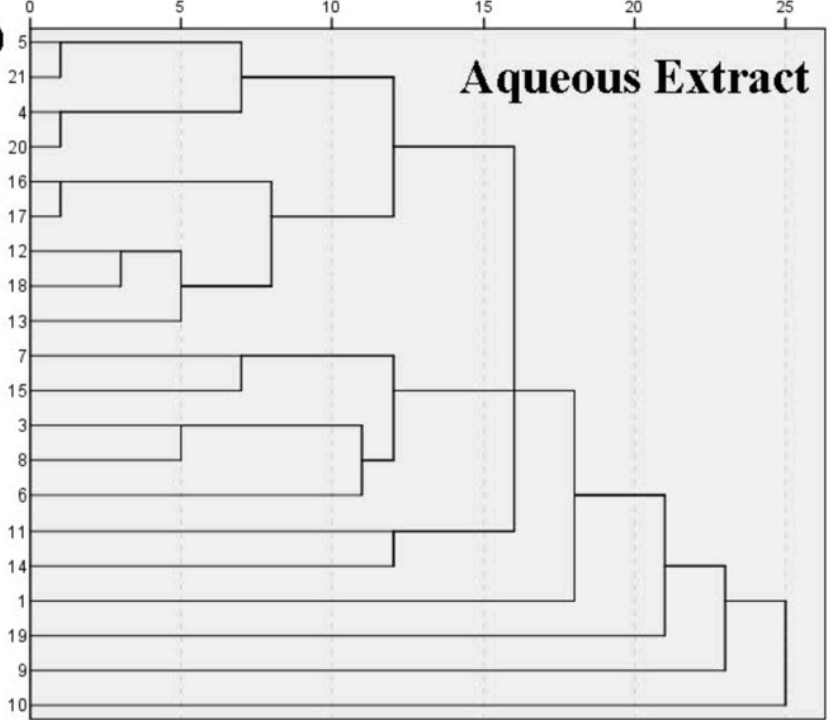

Figure 3 Dendrograms illustrating the average linkage between groups of complexes (hierarchical cluster analysis, rescaled distance cluster combine) for compounds detected by GC-MS in the four extracts (ethanolic, petroleum ether, chloroform and aqueous extracts) of the $21 \mathrm{ST}$ (shammah) samples under study.

with this sample was the presence of arsenous acid tris(trimethylsilyl) ester, a toxic arsenic compound. 1-Tert-butyl-3,3,4trimethyl-3,4-dihydro-1H-benzopyran-1-ol was the benzopyran derivative present. Among siloxanes, dodecamethyl cyclohexasiloxane and hexamethyl cyclotrisiloxane were also present.

The last variety of shammah analyzed was Suhail shammah from Sabya (S21). It also possessed generally detected components and no new compound was identified. Nicotine was present as 7.05-89.68\% of the total components. Alkanes such as tridecane and pentadecane were present in different extracts while tetratetracontane was present as $5.43 \%$ only in aqueous extract. Methyl palmitate was the only fatty ester present in all extracts. Dodecamethyl cyclohexasiloxane and hexamethyl cyclotrisiloxane were the two siloxane derivatives present in different extracts from 0.49 to $4.41 \%$.

To study the relation between the components of different samples, statistical chemometric multivariate analysis, hierarchical cluster analysis (HCA) was performed. The four extracts and the components detected in all the samples were analyzed. For the GC-MS results of ethanolic (Fig. 3A) extract, we could determine that there were four cluster of samples based on the clustering method used. The cluster solution was seen as a sudden jump (gap) in the distance coefficient. The solution before the gap indicated good solution. S1, S2, S3, S4, S5, S7, S11 and $S 14$, were distributed in the same cluster, whereas types S12, S15, S16, S17 and S20 had the similar characteristics to be included in the same cluster. Distribution of the samples was based on their area\% as shown in Fig. 3A.

The cluster analysis for the chloroform extract (Fig. 3C) highlighted five clusters of samples. Samples S2, S3, S4, S5, S6, S7, S11 and S15 were found to be distributed in the same cluster, whereas, S12, S16, S17, S18 and S19 had similar characteristics to be included in the same cluster and samples S5, S9, S14, S20 and S21 were included in the same cluster. Distribution of the samples based on their area\% is shown in Fig. 3C. The cluster analysis results for the petroleum ether and aqueous extracts are shown in Fig. 3 (B and D, respectively).

\section{Discussion}

All the shammah samples were analyzed successfully by a validated method using GC-MS technique. A total of 61 different 
phytocomponents were identified in minor to major percentage in different samples. Out of these, 26 constituents were found to be potentially hazardous to human health (Table 1) that were present in almost all samples signifying their toxic effects in humans. These constituents have been reported to be perilous when consumed in even minute quantities for long.

Nicotine, (3-(1-methyl-2-pyrrolidinyl) pyridine) and its derivative, $((1 \mathrm{~s}, 2 \mathrm{~s})$-nicotine- $\mathrm{N}$-oxide) were identified in all shammah samples included in this study, except Adani Haar (hot) shammah with cardamom from Ahad al Masarihah (S18), which was different from all other samples. The reason for not detecting nicotine in this sample could be attributed to the fact that this product contains no or very little tobacco and instead, they possess other components such as areca nut with flavouring agents.

Highly toxic and even carcinogenic compounds identified in several shammah samples were higher $n$-alkanes such as tridecane, tetradecane, pentadecane, nonacosane, tetratriacontane and dodecane. Tridecane is an asphyxiant (similar to the C6-C10 alkanes) when aspired into the lungs. These alkanes can even cause death slowly and can cause chemical pneumonitis. ${ }^{17}$ Tetradecane is reported to be a carcinogen and tumor promoter in a two-stage experiment of benzo[a]pyrene carcinogenicity model in mice as well as to enhance the mitogenic response of murine spleen lymphocytes to the lectin phytohaemaglutinin. ${ }^{17}$ Pentadecane is harmful if taken by inhalation, ingestion, or even skin absorption as when aspirated into the lungs, it is shown to be an asphyxiant in an in vitro rabbit heart mitochondrial model..$^{18}$ On the other hand, nonacosane, a higher $n$-alkane (containing 29 carbon atoms) is also a known toxic compound. A case report had described human disorder characterized by the accumulation of plant long-chain $n$-alkanes in viscera of a human patient. ${ }^{19}$ Study of $n$-alkane distribution in patient tissues showed a major accumulation in lumbo-aortic lymph nodes, adrenal glands, lung and liver significantly, with lower amounts detected in myocardium and kidney, whereas, non-detectable level was found in brain. ${ }^{19}$ Dodecane can also be irritating to mucous membranes and had been shown previously to be a promoter of skin carcinogenesis for ultraviolet radiation. ${ }^{17}$ Although it did not produce an increase in mutations when tested in the Ames Salmonella typhimurium assay with and without metabolic activation, however, it was able to enhance mutagenesis induced by methylazoxymethanol acetate at the ouabain resistance locus. ${ }^{17}$ Tetratriacontane is also a member of homologous series of $n$-alkanes ranging from $n$-C12- $n$-C 31 and is reported to be accumulated in samples of liver, heart, kidneys, muscle and adipose bovine tissues. ${ }^{20}$

Other potential carcinogenic components detected in shammah samples included fatty acid ester methyl 7-octadecenoate, which is known to promote skin tumor formation in mice. While the fatty acid, octadecenoic acid (stearic acid) is known to produce acute toxicity, DNA damage, mutation, and has tumorigenic effects. ${ }^{17}$ Pyridine,3-(2-pyrrolidinyl) is chemically known to be harmful if swallowed and causes damage and specific target organ toxicity upon single exposure. ${ }^{21}$

Another interesting finding of this study was the determination of $\mathrm{N}$-methoxycarbonyl amphetamine in three Shammah samples i.e. Khadrah shammah (S8) and Arishi shammah (S13) from Abu Arish and Sudani shammah from Sabya (S20). $\mathrm{N}$-Methoxycarbonyl amphetamine is an amphetamine derivative that is classified as the drug of abuse and is associated with numerous dose dependent adverse effects. Upon ingestion, this compound may induce hallucination and accelerated and irregular heartbeat, blurred vision, and a strong feeling of intoxication. At high doses, it may induce nausea and vomiting, severe hyperthermia and hallucinations. ${ }^{22}$ The presence of amphetamine in these shammah samples may be an indicative of adulteration by some other plant products containing amphetamine and having similar properties. This adulteration might be done by the local manufacturers to enhance the organoleptic as well as mood-elevating properties of shammah. Similarly, caffeine was also noticed in some shammah samples including S16, S17 and S19 in minor percentages (0.44-1.41 \%) suggesting the presence of other products such as tea leaves or coffee mixed with these shammah samples. These tea leaves might have some stringent effect on the shammah samples with improved taste and effect. Our study has also shown the presence of arseniccontaining compound arsenous acid tris(trimethylsilyl) ester in S20 shammah samples, which is highly toxic and carcinogenic even in minute quantities. The anhydride form of arsenous acid, arsenic trioxide, is used as a herbicide, pesticide, and rodenticide. Another residual herbicide identified in shammah samples was metazachor (S10; 0.49-3.1\%) which is known to be potential liver toxicant. $^{23}$

Components with lower but considerable degree of toxicity include 1,2-benzenedicarboxylic acid dibutyl ester and hexanedioic acid, which have been reported to produce kidney and testis damage when given to the experimental animals for longer period. ${ }^{24}$ 1,1,1,3,3,5,5-Heptamethyltrisiloxane, which was detected in several shammah samples may cause respiratory irritation and specific target organ toxicity. ${ }^{25}$ Cotinine is also known to be toxic in high doses. ${ }^{26} N, N^{\prime}$-Diethyl urea, oleanitrile, 9-octadecenoic acid-1,2,3-propanetriyl ester, octacosane methyl heptadecanoate are also harmful in case of skin contact (irritant), eye contact (irritant) and in case of ingestion and inhalation.

Hierarchial cluster analysis (HCA) is a multivariate statistical technique to determine a hierarchy of clusters which is graphically presented as a dendrogram or a tree. The distance between the pairs is measured which is a representative of dissimilarity between sets. In dendrograms, the variables are joined together in a hierarchical fashion. The closest are the most similar, whereas, the furthest are the most different. Samples falling in the same cluster have similar characteristics or least dissimilarity and are separated by lesser distances.

\section{Conclusion}

On the basis of above findings, it can be concluded that the shammah samples collected from Jazan province of Saudi Arabia were different in terms of chemical constituents and their concentrations. Shammah is an admixture of several components that is prepared by local manufacturers by adding unknown substances to the tobacco leaves in order to enhance its acceptability and effects. Attempts were made to identify these unknown substances in this study to recognize their additional hazards when ingested by the user. Several unexpected, deleterious and toxic constituents were detected in all shammah samples which together pose serious threats to human health. The identification of the constituents would lead to educate the shammah users regarding the toxicity and would help in the cessation programme. It would also help the local bodies to have a check on the manufacture of these shammah preparations so that the adulteration can be minimized.

\section{Supplementary Material}

Components identified in various shammah samples by GC-MS are listed in Table S1.

\section{List of Abbreviations}

GC-MS: Gas Chromatography-Mass Spectroscopy; ST: smoke- 
less tobacco; HCA: Hierarchical cluster analysis; NNK: nicotine-derived nitrosamine ketone; NNN: $N$-nitrosonornicotine; ROS: reactive oxygen species; NIST08: National Institute of Standards and Technology 08; SPPS: Statistical Package for the Social Sciences

\section{Competing Interests}

The authors declare that they have no competing interests.

\section{Funding}

This work was funded by the Deanship of Scientific Research, Jazan University (Grant no. RG-2-9).

\section{Acknowledgements}

Authors are thankful to the Deanship of Scientific Research, Jazan University, for providing financial assistance (Research Group no. RG-2-9) to carry out this research work.

\section{${ }^{8}$ ORCID iD}

H.A. Alhazmi: (iD orcid.org/0000-0002-9647-9006

\section{References}

1 National Cancer Institute and Centers for Disease Control and Prevention, 2014, Smokeless Tobacco and Public Health: A Global Perspective, US Department of Health and Human Services, Centers for Disease Control and Prevention and National Institutes of Health, National Cancer Institute, Bethesda, MD.

https://cancercontrol.cancer.gov/brp/tcrb/global-perspective/ SmokelessTobaccoAndPublicHealth.pdf (accessed 6 January 2018).

2 R.M. Alsanosy, Smokeless tobacco (shammah) in Saudi Arabia: a review of its pattern of use, prevalence, and potential role in oral cancer, Asian Pac. J. Cancer Prev., 2014, 15, 6477-6483.

3 J.C. Winter, eds., Tobacco use by Native North Americans: Sacred Smoke and Silent Killer, University of Oklahoma Press, Chicago, 2000.

4 M. Valdes de la Cruz, C. Gonzalez and R. Lara, Genetic diversity of wild species of Nicotiana genus I: characterization using biochemical markers, Revista de Protección Vegetal., 2010, 25, 88-97.

5 International Agency for Research on Cancer. Smokeless Tobacco and Some Tobacco-Specific N-Nitrosamines, World Health Organization International Agency for Research on Cancer, Lyon, France, IARC Monographs on the Evaluation of Carcinogenic Risks to Humans, Vol. 89, 2007,

https://monographs.iarc.fr/ENG/recentpub/mono89.pdf (accessed 6 January 2018)

6 N. Al-Mukhaini, T. Ba-Omar, E. Eltayeb, A. Al-Shihi, N. Al-Riyami, J. Al-Belushi and K. Al-Adawi, Liver and kidney toxicity induced by Afzal smokeless tobacco product in Oman, Tissue Cell., 2017, 49, 307-314.

7 M. Liu, J. Jin, H. Pan, J. Feng, C.E. Cerniglia, M. Yang and H. Chen, Effect of smokeless tobacco products on human oral bacteria growth and viability, Anaerobe, 2016, 42, 152-161.

8 J. Sun, J. Jin, R.D. Beger, C.E. Cerniglia, M. Yang and H. Chen, Metabolomics evaluation of the impact of smokeless tobacco exposure on the oral bacterium Capnocytophaga sputigena, Toxicol. in vitro, 2016, 36, 133-141.

9 S. Das, P. Upadhaya and S. Giri, Arsenic and smokeless tobacco induce genotoxicity, sperm abnormality as well as oxidative stress in mice in vivo, Genes Environ., 2016, 38, 4.

DOI: 10.1186/s41021-016-0031-2

10 N. Moghbel, B. Ryu, P.J. Cabot and K.J. Steadman, In vitro cytotoxicity of Nicotiana gossei leaves, used in the Australian aboriginal smokeless tobacco known as Pituri or Mingkulpa, Toxicol Lett., 2016, 254, 45-51.

11 C. Yu, Z. Zhang, Y. Liu, Y. Zong, Y. Chen, X. Du, et al., Toxicity of smokeless tobacco extract after 184-day repeated oral administration in rats, Int. J. Environ. Res. Public Health, 2016, 13, 281. DOI: 3390/ijerph13030281

12 M.A. Song, C. Marian, T.M. Brasky, S. Reisinger, M. Djordjevic and P.G. Shields, Chemical and toxicological characteristics of conventional and low-TSNA moist snuff tobacco products, Toxicol. Lett., 2016, 245, 68-77.

13 S. Biswas, K. Manna, U. Das, A. Khan, A. Pradhan and A. Sengupta, et al., Smokeless tobacco consumption impedes metabolic, cellular, apoptotic and systemic stress pattern: a study on government employees in Kolkata, India, Sci. Rep., 2015, 5, 18284. DOI: $10.1038 /$ srep18284

14 P. Roy, A. Mukherjee and S. Giri, Evaluation of genetic damage in tobacco and arsenic exposed population of southern Assam, India using buccal cytome assay and comet assay, Ecotoxicol. Environ. Saf., 2016, 124, 169-176.

15 S.S. Arain, T.G. Kazi, H.I. Afridi, F.N. Talpur, A.G. Kazi and K.D. Brahman, et al., Estimation of nickel in different smokeless tobacco products and their impact on human health of oral cancer patients, Nutr. Cancer, 2015, 67, 1063-1074.

16 K. Niaz, F. Maqbool, F. Khan, H. Bahadar, I.F. Hassan and M. Abdollahi, Smokeless tobacco (paan and gutkha) consumption, prevalence, and contribution to oral cancer, Epidemiol. Health, 2017, 9, 39. DOI: 10.4178/epih.e2017009

17 E. Bingham, B. Cohrssen and C.H. Powell, Patty's Toxicology, 3rd edn., John Wiley \& Sons, New York, 2001.

18 A.R. Borgatti, G. Trigari, V. Ventrella and A. Pagliarani, Interaction of $n$-alkanes with respiration and oxidative phosphorylation in rabbit heart mitochondria: $n$-dodecane, $n$-pentadecane and $n$-octadecane, Boll. Soc. Ital. Biol. Sper. 1981, 57, 1583-1589.

19 R. Salvayre, A. Nègre, F. Rocchiccioli, C. Duboucher, A. Maret and C. Vieu, et al., A new human pathology with visceral accumulation of long-chain $n$-alkanes, tissue distribution of the stored compounds and pathophysiological hypotheses, Biochim. Biophys. Acta, 1988, 958, 477-483.

20 C. Lintas, A.M. Balduzzi, M.P. Bernardini and A. Di Muccio, Distribution of hydrocarbons in bovine tissues, Lipids, 1979, 14, 298-303.

21 Chemical Book CAS database list, http://www.chemicalbook.com/ChemicalProductProperty_EN_CB4 385206.htm (accessed 28 March 2018).

22 G. Smets, K. Bronselaer, K. De Munnynck, K. De Feyter, W. Van de Voorde and M. Sabbe, Amphetamine toxicity in the emergency department, Eur. J. Emerg. Med., 2005, 12, 193-197.

23 PPDB: Pesticide Properties Data Base. https://sitem.herts.ac.uk/aeru/ppdb/en/Reports/450.htm (accessed 28 March 2018)

24 V.O. Sheftel, Indirect Food Additives and Polymers, Migration and Toxicology, Lewis Publishers, Boca Raton, FL, 2000.

25 PubChem Substance and Compound database.

https://pubchem.ncbi.nlm.nih.gov/compound/1 11 1 $1 \begin{array}{lllll}3 & 5 & 5 & 5 & 5\end{array}$ Heptamethyltrisiloxane (accessed 26 March 2018).

26 T. Jacob, N. Clouden, A. Hingorani and E. Ascher, The effect of cotinine on telomerase activity in human vascular smooth muscle cells, J. Cardiovasc. Surg., 2009, 50, 345-349. 


\section{Supplementary material to:}

H.A. Alhazmi, A. Khalid, S. Sultana, S.I. Abdelwahab, W. Ahsan, M.E. Oraiby and M. Al Bratty, Determination of Phytocomponents of Twenty-one Varieties of Smokeless Tobacco using Gas Chromatography-Mass Spectroscopy (GC-MS),

S. Afr. J. Chem., 2019, 72, 47-54. 
Table S1 All Components Identified in Various Shammah Samples by GC-MS.

\begin{tabular}{|c|c|c|c|c|c|c|c|c|c|c|c|c|c|c|c|c|c|c|c|c|c|c|c|c|c|c|c|c|c|c|c|c|c|c|c|c|c|c|c|c|}
\hline & & 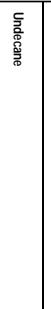 & 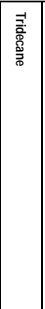 & 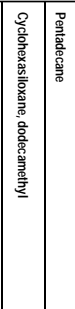 & 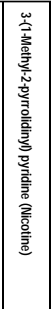 & 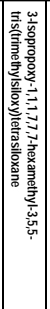 & & 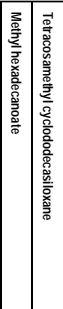 & 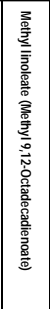 & 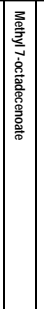 & 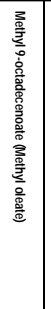 & 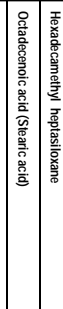 & & & 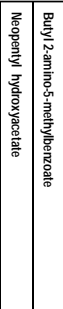 & 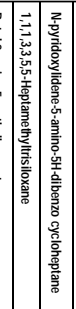 & 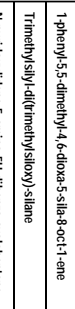 & 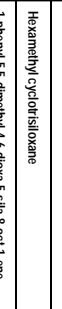 & 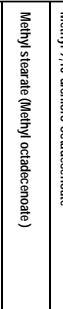 & 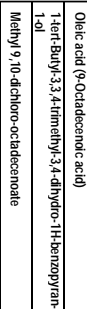 & & & 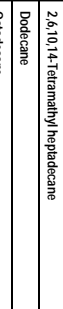 & & 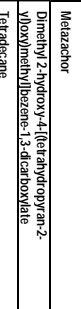 & 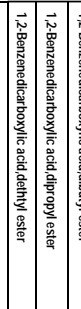 & 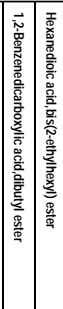 & 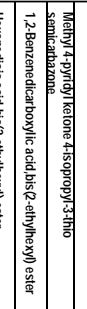 & 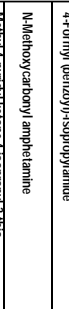 & 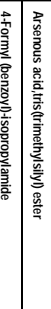 & 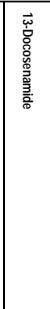 & 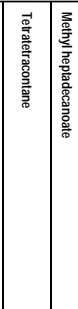 & 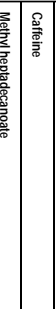 & 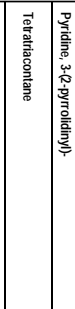 & 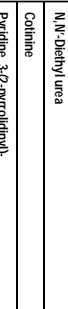 & 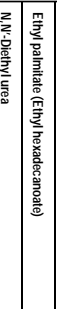 & 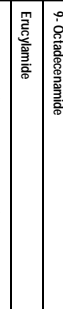 & 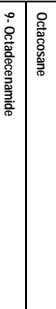 & 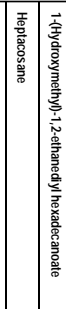 & 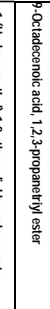 \\
\hline \multirow{5}{*}{ 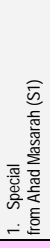 } & $\begin{array}{l}\begin{array}{l}\text { Retention } \\
\text { itime }\end{array} \\
\end{array}$ & 4.19 & \begin{tabular}{|l|}
4.21 \\
\end{tabular} & \begin{tabular}{|l|l|l}
6.05 \\
\end{tabular} & \begin{tabular}{|c|}
6.58 \\
\end{tabular} & \begin{tabular}{|c|}
7.8 \\
\end{tabular} & 7.71 & \begin{tabular}{|c|c|c|}
0 & 11.5 \\
98 & 4 \\
\end{tabular} & \begin{tabular}{|l|}
12.09 \\
\end{tabular} & \begin{tabular}{|r|}
21.1 \\
3 \\
\end{tabular} & 12.14 & \begin{tabular}{|c|c|c|c|}
2.2 & 13. \\
8
\end{tabular} & $\begin{array}{c}3.4 \\
\begin{array}{c}3.4 \\
6\end{array} \\
6\end{array}$ & 414.4 & \begin{tabular}{|r|r|r}
16.1 & 16.3 \\
\end{tabular} & \begin{tabular}{r|r|r|r|r|}
166 & 18.6 \\
\end{tabular} & \begin{tabular}{|c|c|c|c|c|}
19.7 & 21.3 \\
\end{tabular} & \begin{tabular}{|l|l|l|l|l|}
9 & 24,1 \\
\end{tabular} & & & & & & & & & & & & & & & & & & & & & & \\
\hline & \begin{tabular}{|l}
$\begin{array}{l}\text { Area\%\% Pets } \\
\text { enther }\end{array}$ \\
ets
\end{tabular} & & \begin{tabular}{|l|}
0.4 \\
\end{tabular} & \begin{tabular}{|l|l|l|}
0.51 & 1.08 \\
\end{tabular} & \begin{tabular}{|l|}
66.28 \\
\end{tabular} & \begin{tabular}{|l|}
0.37 \\
\end{tabular} & & \begin{tabular}{|l|l|}
12 & - \\
66 & \\
\end{tabular} & \begin{tabular}{|l|}
1.75 \\
\end{tabular} & - & \begin{tabular}{ll|}
12.63 \\
\end{tabular} & 1.3 & - & $-\cdots$ & \begin{tabular}{|l|l|}
- & - \\
\end{tabular} & 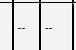 & \begin{tabular}{|l|l}
- & - \\
\end{tabular} & 7 & & & & & & & & & & & & & & & & & & & & & & \\
\hline & \begin{tabular}{|l}
$\begin{array}{l}\text { Area\% } \\
\text { Chloroform }\end{array}$ \\
\end{tabular} & 1.6 & & \begin{tabular}{|l|l|}
1.06 & 1.03 \\
\end{tabular} & 2.1 & \begin{tabular}{|l|}
1.19 \\
\end{tabular} & & \begin{tabular}{|r|}
0.9 \\
5
\end{tabular} & - &. & 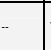 & 1.61 -- & - & \begin{tabular}{|r|}
10.9 \\
5
\end{tabular} & \begin{tabular}{|l|l|l|}
2.6 & 2.31 \\
\end{tabular} & \begin{tabular}{l|l|}
3.1 & 6.17 \\
4
\end{tabular} & \begin{tabular}{|l|l|l|l}
7.57 & 4.13 \\
\end{tabular} & \begin{tabular}{|l|l|l|}
13 & 3.76 \\
\end{tabular} & & & & & & & & & & & & & & & & & & & & & & \\
\hline & \begin{tabular}{|l|} 
Area\% \\
Enananol
\end{tabular} & 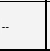 & \begin{tabular}{|l|l|} 
\\
\end{tabular} & 2.79 & \begin{tabular}{|l|}
67.84 \\
\end{tabular} & 2.02 & 0.8 & \begin{tabular}{|r|}
9.9 \\
7.
\end{tabular} & 1.05 & 9.85 & 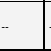 & $\ldots$ & 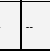 & $\ldots$ & \begin{tabular}{|l|l|}
- & - \\
\end{tabular} & \begin{tabular}{|l|l|}
- & - \\
\end{tabular} & \begin{tabular}{|l|l|} 
& - \\
\end{tabular} & & & & & & & & & & & & & & & & & & & & & & & \\
\hline & \begin{tabular}{|l}
$\begin{array}{l}\text { Area\% } \\
\text { Aqueous }\end{array}$ \\
\end{tabular} & 0.23 & - & \begin{tabular}{|l|l}
0.35 & - \\
\end{tabular} & \begin{tabular}{|c|}
33.38 \\
\end{tabular} & \begin{tabular}{|l|}
0.29 \\
\end{tabular} & 1.02 & \begin{tabular}{|r|r}
1.4 & 3.68 \\
\end{tabular} & \begin{tabular}{|l|}
0.18 \\
\end{tabular} & & - & $\approx \begin{array}{l}0.3 \\
4\end{array}$ & $\begin{array}{l}0.3 \\
40.8 \\
\end{array}$ & & \begin{tabular}{|l|l}
- & - \\
\end{tabular} & 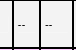 & \begin{tabular}{|l|l}
- & - \\
\end{tabular} & & & & & & & & & & & & & & & & & & & & & & & \\
\hline \multirow{5}{*}{ 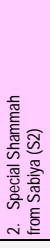 } & $\begin{array}{l}\begin{array}{l}\text { Retention } \\
\text { time }\end{array} \\
\end{array}$ & 4.19 & \begin{tabular}{|l|l|} 
\\
\end{tabular} & \begin{tabular}{|l|l}
6.1 & 6.1 \\
\end{tabular} & $\begin{array}{l}6.58 \\
\end{array}$ & $\begin{array}{l}.48 \\
\end{array}$ & & \begin{tabular}{|l|}
10 \\
98 \\
\end{tabular} & 12.09 & & 12.13 & & & & & & & & & $\begin{array}{r}14.4 \\
6 \\
\end{array}$ & $\begin{array}{r}16.9 \\
1 \\
\end{array}$ & & 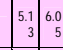 & 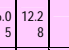 & & & & & & & & & & & & & & & & \\
\hline & $\begin{array}{l}\begin{array}{l}\text { Area\% Pet. } \\
\text { ether }\end{array} \\
\text {. }\end{array}$ & & \begin{tabular}{|l|}
.88 \\
\end{tabular} & 3.06 - & \begin{tabular}{|l|} 
\\
\end{tabular} & \begin{tabular}{|l|}
1.69 \\
\end{tabular} & & $\begin{array}{r}9.5 \\
4 \\
\end{array}$ & 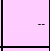 & & 6.45 & & & & & & & & & . & - & & \begin{tabular}{|l|l}
1.4 .7 \\
4 \\
\end{tabular} & 7. & & & & & & & & & & & & & & & & \\
\hline & 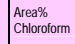 & 1.6 & 3.13 & \begin{tabular}{|l|l|}
1.98 & 1.45 \\
\end{tabular} & 28.71 & 1.74 & & $\left|\begin{array}{|c|}28 . \\
28\end{array}\right|$ & 3.4 & & 27.25 & & & & & & & & & - & - & & 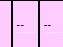 & 1.98 & & & & & & & & & & & & & & & & \\
\hline & $\begin{array}{l}\text { Area\% } \\
\text { Ethanol }\end{array}$ & 1 & \begin{tabular}{|l|l|}
1.33 \\
\end{tabular} & \begin{tabular}{|c|c|}
0.62 & -
\end{tabular} & \begin{tabular}{|l|}
23.41 \\
\end{tabular} & \begin{tabular}{|l|}
0.33 \\
\end{tabular} & & $\mid$\begin{tabular}{|l|}
34 \\
66 \\
66
\end{tabular} & \begin{tabular}{|l|}
2.58 \\
\end{tabular} & & 17.86 & & & & & & & & & 8.12 & 1.63 & &.- & 3.19 & & & & & & & & & & & & & & & & \\
\hline & $\begin{array}{l}\text { Area\% } \\
\text { Aqueous }\end{array}$ & 0.23 & \begin{tabular}{|l|l|}
1.53 \\
\end{tabular} & \begin{tabular}{|l|l|}
1.23 & 0.52 \\
\end{tabular} & 15.77 & \begin{tabular}{|l|}
0.87 \\
\end{tabular} & & \begin{tabular}{|l|}
33 \\
62 \\
\end{tabular} & 4.5 & & 35.26 & & & & & & & & & . & 1.39 & & \begin{tabular}{|l|l|l|l|l|l|} 
\\
6
\end{tabular} & 28. & & & & & & & & & & & & & & & & \\
\hline \multirow{5}{*}{ 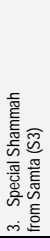 } & $\begin{array}{l}\begin{array}{l}\text { Retention } \\
\text { time }\end{array} \\
\text { int }\end{array}$ & 4.19 & 6.06 & \begin{tabular}{|l|}
6.01 \\
\end{tabular} & $\begin{array}{l}6.58 \\
\end{array}$ & 7.48 & & $\mid$\begin{tabular}{|}
10 \\
99 \\
\end{tabular} & \begin{tabular}{|l|}
2.09 \\
\end{tabular} & & 12.13 & & & & & & & & \begin{tabular}{|l|}
12.28 \\
\end{tabular} & & & & & 4.2 & 42 & & & & & & & & & \begin{tabular}{|l|}
17.57 \\
\end{tabular} & & & & & & \\
\hline & $\begin{array}{l}\text { Area\% Pet } \\
\text { ether }\end{array}$ & 0.62 & & \begin{tabular}{|l|}
3.57 \\
\end{tabular} & \begin{tabular}{|l|}
88.81 \\
\end{tabular} & \begin{tabular}{|l|}
1.31 \\
\end{tabular} & & \begin{tabular}{|c|}
0.7 \\
9 \\
\end{tabular} & 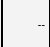 & & . & & & & & & & & - & & & & & 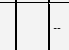 & . & & & & & & & & & - & & & & & & \\
\hline & \begin{tabular}{|l}
$\begin{array}{l}\text { Area\% } \% \\
\text { Chloroform }\end{array}$ \\
\end{tabular} & 1.54 & 0.42 & \begin{tabular}{|l|l|}
3.16 \\
\end{tabular} & \begin{tabular}{|l|}
34.74 \\
\end{tabular} & \begin{tabular}{|l|l|} 
\\
\end{tabular} & & \begin{tabular}{|c|}
22 \\
96 \\
\end{tabular} & 3.81 & & 23.38 & & & & & & & & 1.92 & & & & & .. & . & & & & & & & & &. & & & & & & \\
\hline & $\begin{array}{l}\text { Area\% } \\
\text { Ethanol }\end{array}$ & 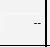 & 0.81 & \begin{tabular}{|l|} 
\\
\end{tabular} & 11.11 & & & \begin{tabular}{|r|}
7.2 \\
2 \\
\end{tabular} & $\ldots$ & & 5 & & & & & & & & 0.89 & & & & & ${ }^{2.1}$ & ?.1. & & & & & & & & & - & & & & & & \\
\hline & \begin{tabular}{|l} 
Area\% \\
Aqueous
\end{tabular} & \begin{tabular}{|l|}
0.16 \\
\end{tabular} & \begin{tabular}{|l|l|} 
\\
\end{tabular} & \begin{tabular}{|l|}
0.04 \\
\end{tabular} & \begin{tabular}{|l|}
46.12 \\
\end{tabular} & & & \begin{tabular}{|r|}
18 \\
6
\end{tabular} & 4.31 & & 20.16 & & & & & & & & 1.85 & & & & & $\begin{array}{l}0.4 \\
2\end{array}$ & 8 & & & & & & & & & 2.92 & & & & & & \\
\hline \multirow{5}{*}{ 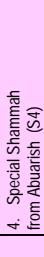 } & \begin{tabular}{|l|}
$\begin{array}{l}\text { Retention } \\
\text { time }\end{array}$ \\
\end{tabular} & 4.19 & & \begin{tabular}{|l|l|}
6.01 \\
\end{tabular} & \begin{tabular}{|l|l|}
6.58 \\
\end{tabular} & & & \begin{tabular}{|c|}
10. \\
99 \\
\end{tabular} & 12.09 & & 12.13 & & $\begin{array}{l}14,2 \\
2^{14.2} \\
\end{array}$ & & & & & & \begin{tabular}{|l|l|}
12.28 \\
\end{tabular} & & ${ }_{2}^{15.5}$ & 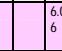 & & & & & & & & & & & & $\begin{array}{l}17.57 \\
\end{array}$ & & & $\begin{array}{l}10.0 \\
6.0 \\
6\end{array}$ & 76.0 & & \\
\hline & $\begin{array}{l}\begin{array}{l}\text { Area\%" Pett } \\
\text { ether }\end{array} \\
\text { ether }\end{array}$ & 0.49 & & \begin{tabular}{|l|}
3.2 \\
\end{tabular} & \begin{tabular}{|l|l|}
88.08 \\
\end{tabular} & & & $\begin{array}{r}2.0 \\
8 \\
\end{array}$ & & & ${ }^{1.49}$ & & & & & & & & & & & {$\left[\begin{array}{l}0.5 \\
9\end{array}\right.$} & & & & & & & & & & & & & & & & & & \\
\hline & $\begin{array}{l}\begin{array}{l}\text { Area\% \% } \\
\text { Chloroform }\end{array} \\
\end{array}$ & $\begin{array}{l}1.64 \\
\end{array}$ & & \begin{tabular}{|l|l|l|} 
& \\
\end{tabular} & \begin{tabular}{|l|l|}
27.18 \\
\end{tabular} & & & \begin{tabular}{|c|}
$\begin{array}{c}21 \\
78\end{array}$ \\
\end{tabular} & 3.05 & & 20.16 & & & & & & & & & & & $\begin{array}{l}0.6 \\
3 \\
3\end{array}$ & & & & & & & & & & & & & & & & & & \\
\hline & $\begin{array}{l}\begin{array}{l}\text { Area\% } \\
\text { EEnarol }\end{array} \\
\end{array}$ & & & 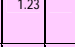 & $\mid$ & & & $7^{7.5}$ & & & 5.84 & & & & & & & & 0.75 & & 1.5 & & & & & & & & & & & & & & & & & & & \\
\hline & $\begin{array}{l}\text { Area\% } \\
\text { Aqueous }\end{array}$ & & & & 1228 & & & . & & & & & & & & & & & & & & & & & & & & & & & & & & & & & & & & \\
\hline & & & & & & & & & & & & & & & & & & & & & & & & & & & & & & & & & & & & & & & & \\
\hline & & & & & & & & & & & & & & & & & & & & & & & & & & & & & & & & & & & & & & & & \\
\hline
\end{tabular}





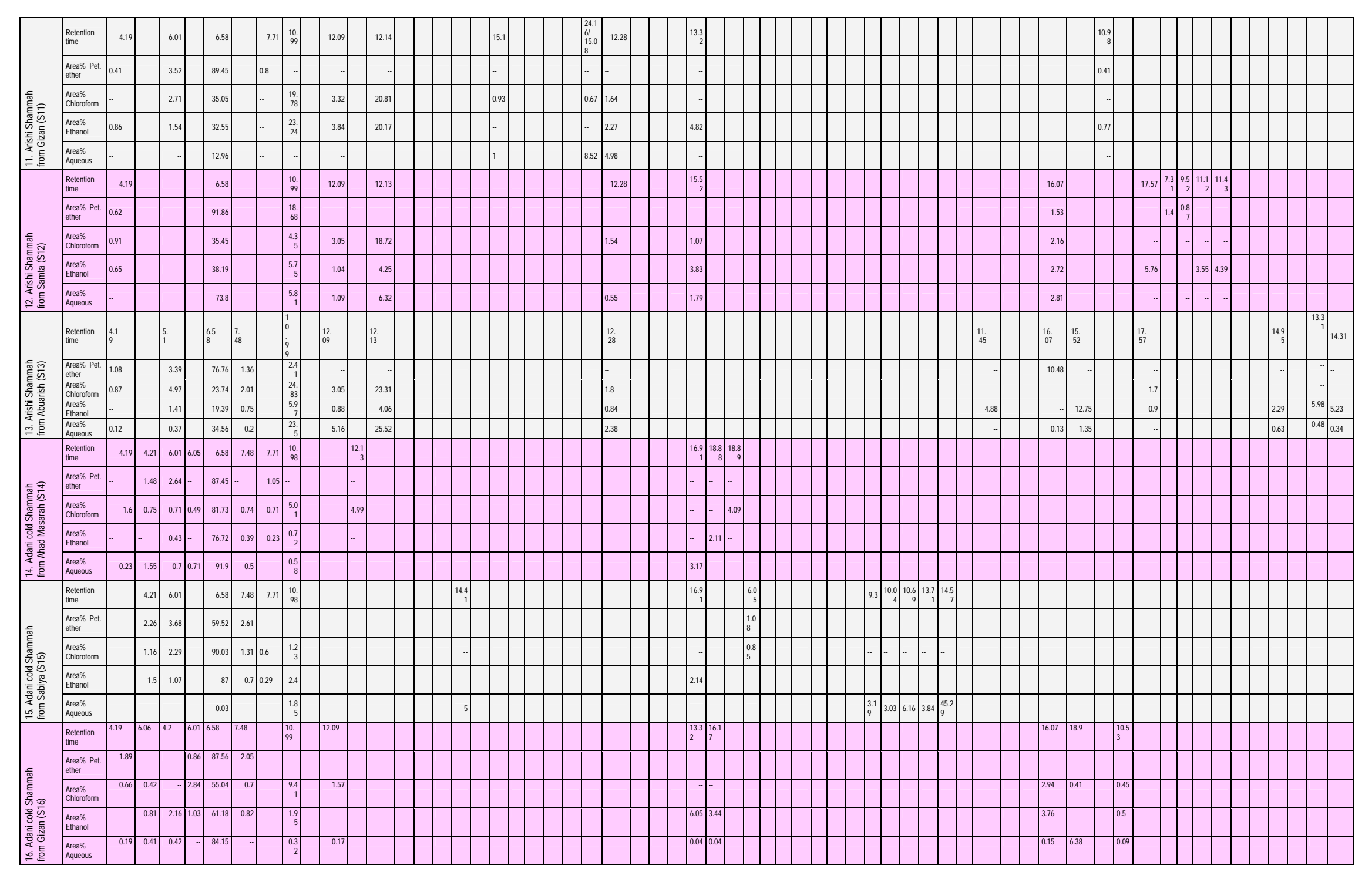




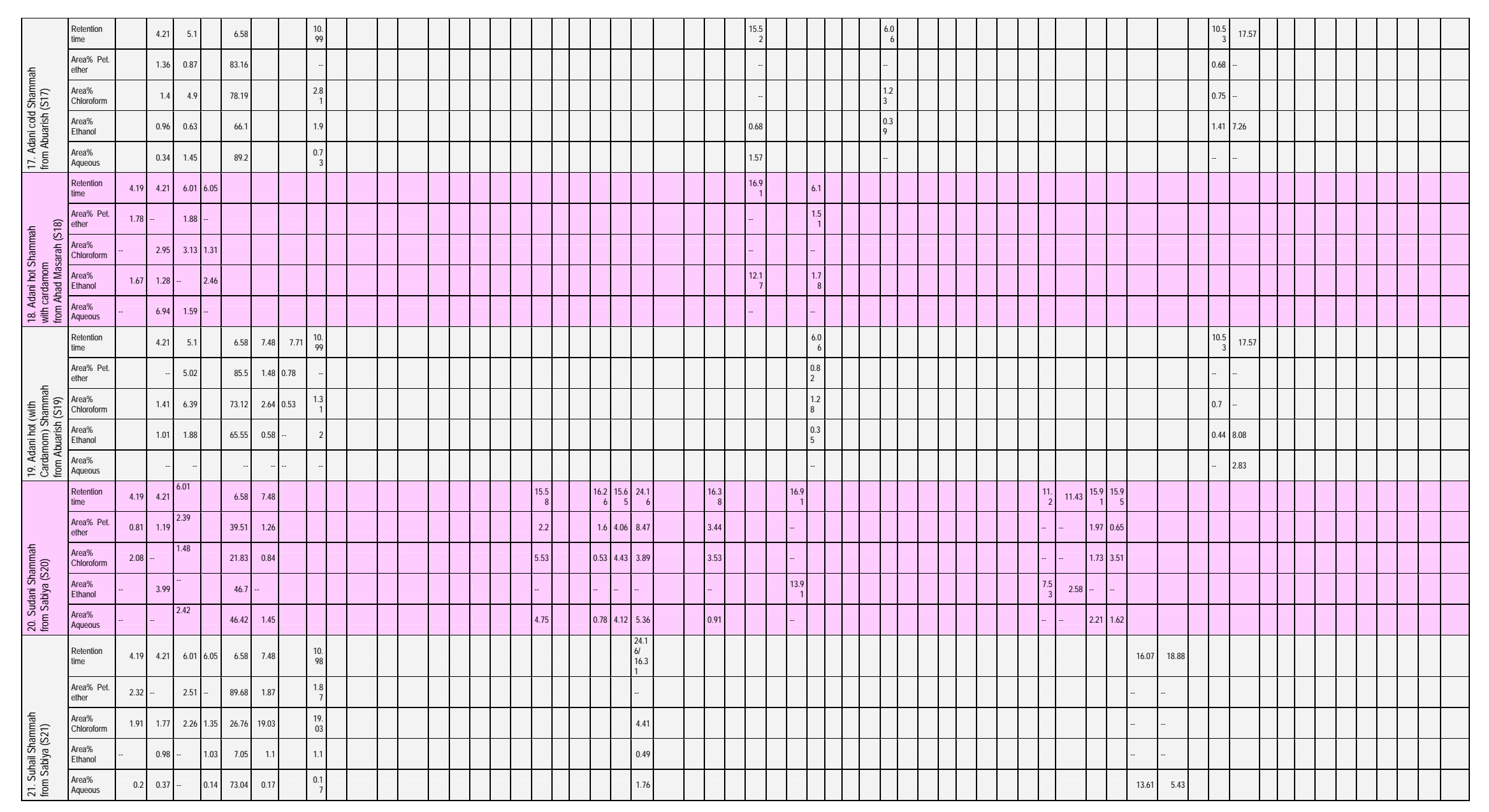

